Carla Vitola Gonchalves ${ }^{\uparrow}$

RaUl ANDRES Mendoza-SASSI ${ }^{1}$

Juraci Almeida Cesar ${ }^{1}$

Natála BolbadILHA de CAstro²

ANa PAULA BORTOLOMEDI ${ }^{2}$

Artigo Original

\author{
Palavras-chave \\ Cuidado pré-natal \\ Índice de massa corporal \\ Gravidez de alto risco \\ Parto \\ Peso ao nascer \\ Keywords \\ Prenatal care \\ Body mass index \\ Pregnancy, high-risk \\ Parturition \\ Birth weight
}

\section{Índice de massa corporal e ganho de peso gestacional como fatores preditores de complicações e do desfecho da gravidez}

\author{
Body mass index and gestational weight gain as factors predicting \\ complications and pregnancy outcome
}

\section{Resumo}

OBJETIVO: Avaliar o impacto do índice de massa corporal (IMC) no início da gestação e do ganho de peso no desfecho gestacional, para que esta medida possa ser implantada e valorizada pelos serviços de saúde de pré-natal. MÉTODO: Estudo transversal de base populacional. Na pesquisa, foram incluídos todos os nascimentos ocorridos nas duas únicas maternidades do município do Rio Grande (RS), em 2007. Dentre as 2.557 puérperas entrevistadas, o cálculo do IMC só pôde ser realizado em 1.117 puérperas. A análise foi realizada no programa Stata 1 1. Nos desfechos hipertensão, diabetes mellitus, trabalho de parto prematuro e cesárea foi realizada regressão logística. No caso do peso ao nascer, o ajuste ocorreu pela regressão logística multinomial, tendo como categoria base o grupo de 2.500 a $4.000 \mathrm{~g}$. Em todas as análises foi adotado valor $p<0,05$ de um teste bicaudal. RESULTADOS: Não foi observado aumento do risco de hipertensão e diabetes nas pacientes dos diferentes grupos de IMC e ganho de peso. $\bigcirc$ risco de parto pré-termo foi evidenciado no grupo com ganho de peso $\leq 8 \mathrm{~kg}(\mathrm{p}<0,05)$. Em relação à via de parto, observa-se uma tendência, quanto maiores o IMC no início da gestação e o ganho de peso durante a gestação, maior o risco de parto cirúrgico, chegando $11 \%$ no grupo de obesas $(p=0,004)$ e a $12 \%$ no grupo com ganho $\geq 17 \mathrm{~kg}(\mathrm{p}=0,001)$. $\bigcirc$ peso do recém-nascido foi influenciado pelo IMC e pelo ganho de peso, sendo que quanto maiores o IMC no início da gestação e o ganho de peso gestacional, maior o risco de macrossomia. CONCLUSÕES: $O$ monitoramento do IMC e o ganho ponderal durante a gestação é procedimento de baixo custo e de grande utilidade para o estabelecimento de intervenções nutricionais visando à redução de riscos maternos e fetais.

\section{Abstract}

PURPOSE: To evaluate the impact of body mass index (BMI) at the beginning of pregnancy and weight gain on pregnancy outcome so that this measure can be implemented and valued by prenatal care health services. METHOD: Crosssectional population-based study of all births in the only two hospitals in Rio Grande city (Brazil), in 2007. Among the 2,557 mothers interviewed, it was possible to calculate BMI in only 1,1 17. The Stata 11 software was used for data analysis. Logist regression was applied to the outomes involving diabetes mellitus, premature labor and cesarean section. Regarding birth weight, data were adjusted by multinomial logistic regression using as base category the group of 2,500 to 4,000 g. The level of significance was set at p-value $<0.05$ in a two-tailed test. RESULTS: There was no increased risk of hypertension or diabetes in patients in the different groups of BMl and weight gain. The risk of preterm delivery was evident in the group with a weight gain $\leq 8 \mathrm{~kg}(\mathrm{p}<0.05)$. Regarding the route of delivery, it was observed that the higher the BMI in early pregnancy $(p=0.001)$ and the greater the weight gain during pregnancy $(p=0.004)$, the greater the risk of surgical delivery, which reached $11 \%$ in the group of obese mothers $(p=0.004)$ and $12 \%$ in the group with a weight gain $\geq 17 \mathrm{~kg}(\mathrm{p}=0.001)$. The weight of the newborns was influenced by BMl and weight gain, and the higher the BMl in early pregnancy and the gestational weight gain, the greater the risk of macrosomia. CONCLUSION: The monitoring of BMI and weight gain during pregnancy is a low cost and useful procedure for the establishment of nutritional interventions aimed at reducing maternal and fetal risks.
Correspondência

Carla Vitola Goncalves Faculdade de Medicina da Universidade Federal do Rio Grande Rua General Osório, $s / n-4^{\circ}$ andar - Centro

CEP: $96201-900$

Rio Grande (RS), Brasil

Recebido

$04 / 04 / 2012$
Trabalho realizado na Faculdade de Medicina da Universidade Federal do Rio Grande - FURG - Rio Grande (RS), Brasil.

' Faculdade de Medicina da Universidade Federal do Rio Grande - FURG - Rio Grande (RS), Brasil.

${ }^{2}$ Curso de Graduação em Medicina da Universidade Federal do Rio Grande - FURG - Rio Grande (RS), Brasil.

Conflito de interesses: não há 
Introdução

A obesidade é um dos maiores problemas de saúde publica no mundo moderno, tanto em países desenvolvidos $^{1-3}$ como nos em desenvolvimento $^{1,4}$. Quando comparados aos indivíduos com peso normal, os com sobrepeso mostram maior risco de desenvolver diabetes mellitus (DM), dislipidemia e hipertensão arterial (HAS), condições que favorecem o desenvolvimento de doenças cardiovasculares (DCV) $)^{2,5}$.

Em relação ao período gestacional, o maior risco para complicações são para as gestantes obesas, que estão suscetíveis a diabetes, hipertensão, parto cirúrgico, enquanto seus filhos são mais propensos a apresentarem macrossomia, riscos de malformação fetal e maior mortalidade perinatal $^{6-17}$. No entanto o baixo peso materno também aumenta os riscos de desfechos desfavoráveis para a mãe e para o concepto, entre os quais pode ser citada a restrição de crescimento intrauterino e a prematuridade ${ }^{6,7,18,19}$.

Segundo a Organização Mundial da Saúde $(\mathrm{OMS})^{20}$, o monitoramento do ganho ponderal durante a gestação é um procedimento de baixo custo e de grande utilidade para o estabelecimento de intervenções nutricionais visando à redução de riscos maternos e fetais. A orientação nutricional pode proporcionar um ganho de peso adequado, prevenindo o ganho excessivo ou diagnosticando o ganho ponderal insuficiente. $\mathrm{O}$ Institute of Medicine dos Estados Unidos (IOM-EUA) reconhece o peso pré-gestacional como um dos principais determinantes do ganho ponderal, recomendando que o ganho de peso ideal seja avaliado em função do estado nutricional inicial da gestante, sendo este definido de acordo com as categorias de índice de massa corporal (IMC) ${ }^{21}$.

O IMC vem sendo um importante instrumento na avaliação do estado nutricional de adultos. Esse índice é calculado pela divisão do valor da massa corporal em quilogramas pelo quadrado da estatura em metros $\left(\mathrm{IMC}=\mathrm{kg} / \mathrm{m}^{2}\right)$. Apesar de haver algumas limitações quanto ao seu uso, o IMC é largamente utilizado por vários motivos: a alta correlação com a massa corporal e indicadores de composição corporal; a capacidade de predizer riscos de patologias; e tornar dispensável o uso de dados de referência antropométricos no diagnóstico do estado nutricional. Para isto é, utiliza-se um ponto de corte fixo ao invés de um valor de distribuição populacional (por exemplo, um indivíduo com IMC > 30 apresenta um determinado risco relacionado à obesidade, ou seja, trata-se de um índice simples e conveniente, para o qual há muitos bancos de dados epidemiológicos disponíveis. De qualquer modo, tirando-se os extremos da magreza e excesso de peso, observados em alguns segmentos da população (atletas e/ou trabalhadores que desenvolvem grande massa muscular), o IMC parece válido como indicador do estado nutricional para a maioria dos indivíduos ${ }^{20}$.
A OMS, o IOM, The Society of Obstetricians and Gynaecologists of Canada (SOCG) e o Ministério da Saúde do Brasil $^{22}$ recomendam ganhos de peso diferenciados de acordo com o estado nutricional pré-gestacional ${ }^{9,20-22}$. Mulheres que apresentam baixo peso devem ter um ganho ponderal de 12,5 a $18,0 \mathrm{~kg}$; gestantes de peso adequado de 11,5 a $16,0 \mathrm{~kg}$; mulheres com sobrepeso de 7,0 a $11,0 \mathrm{~kg}$; e gestantes obesas devem apresentar ganho menor ou igual a $7,0 \mathrm{~kg}$.

Como o estado nutricional pré-gestacional e o ganho ponderal têm implicação direta na saúde materna-infantil, objetivamos com este estudo avaliar o impacto do IMC pré-gestacional e do ganho de peso no desfecho gestacional, para que esta medida possa ser implantada e valorizada pelos serviços de saúde que realizem pré-natal.

\section{Métodos}

Este estudo foi realizado no município do Rio Grande que possui cerca de 200 mil habitantes e é localizado na planície costeira Sul do estado do Rio Grande do Sul. Sua economia é bastante diversificada e o seu produto interno bruto (PIB) per capitalano é de aproximadamente $\mathrm{R} \$ 19.000,00$. No quesito desenvolvimento, ocupa a $29^{\mathrm{a}}$ posição dentre os 453 municípios do estado, com um o Índice de Desenvolvimento Humano (IDH) de 0,793. A sua rede de saúde é constituída por 32 unidades básicas, 2 hospitais gerais e 5 ambulatórios. Pelo menos 99\% dos partos ocorrem em nível hospitalar. Apesar do coeficiente de mortalidade infantil ter declinando nos últimos anos, chegando a 16/1.000, ainda é superior à média do estado, com cerca de 13/1.000 23 .

O delineamento utilizado para o estudo foi do tipo transversal de base populacional. Na pesquisa, foram incluídos todos os nascimentos ocorridos nas duas únicas maternidades do município (Santa Casa de Misericórdia de Rio Grande e Hospital Universitário da Universidade Federal do Rio Grande - FURG) entre $1^{\circ}$ de janeiro e 31 de dezembro de 2007. Estes recém-nascidos deveriam ter peso igual ou superior a 500 gramas ou 20 semanas ou mais de idade gestacional, sendo que suas mães deveriam residir na área urbana ou rural do município.

Dez entrevistadoras foram treinadas para atuar neste estudo. Destas, oito eram acadêmicas do Curso de Medicina da FURG e as outras eram graduadas em Serviço Social. Este treinamento consistiu da leitura do questionário e do manual de instruções, na sua aplicação entre duplas e perante o grupo de entrevistadores. O estudo piloto foi realizado nas duas maternidades da cidade com o objetivo de testar o questionário a ser utilizado, bem como avaliar o tempo necessário à realização da entrevista.

Estes entrevistadores visitavam diariamente as duas maternidades da cidade, que distam não mais que cem metros 
uma da outra. Todas as informações deste estudo foram coletadas com uso de questionário único pré-codificado, com as mães sendo entrevistadas na maternidade em até 24 horas após o parto. Este questionário investigou características demográficas, nível socioeconômico e assistência recebida durante a gestação e o parto.

A identificação das gestantes era feita pela informação obtida diretamente junto à gestante e confirmada através do livro de registro de nascimento de cada maternidade. As parturientes residentes no município do Rio Grande recebiam explicações sobre os objetivos do estudo e convite para participar. Havendo concordância, um termo de consentimento era assinado pela gestante autorizando a realização da entrevista. Ao final de cada dia de trabalho, o entrevistador codificava os questionários por ele aplicados e, no dia seguinte, os entregava na sede do estudo onde os questionários eram revisados e entregues à digitação.

Dentre as 2.557 puérperas entrevistadas, apenas 1.235 tinham informação referente ao peso no início e no final do pré-natal. Portanto, o ganho de peso durante o período gestacional foi avaliado apenas neste grupo. Quanto ao cálculo do IMC, este só foi possível ser realizado em 1.117 puérperas. Além do IMC e do ganho de peso, outras variáveis independentes como idade, cor, ter companheiro, escolaridade e renda per capita foram analisadas como fatores de confusão. Quanto ao desfecho gestacional, os dados referentes à hipertensão e o diabetes mellitus seguiram o relato da paciente. Os dados referentes a trabalho de parto prematuro, tipo de parto e o peso do recém-nascido ao nascer foram coletados do registro médico.

A digitação dos questionários era duplamente realizada por digitadores independentes, em ordem inversa ao do primeiro, no programa Epi-Info 6.04. Ao término da digitação de cada lote, realizava-se comparação das digitações. A análise estatística consistiu na descrição da amostra estudada, seguido de uma análise ajustada para idade, cor, ter companheiro, escolaridade, renda e os cinco desfechos de interesse e cálculo das razões de $0 d d s$. Nos desfechos hipertensão, diabetes mellitus, trabalho de parto prematuro e cesárea (dicotômicos), o ajuste foi realizado mediante a utilização da regressão logística. No caso do peso ao nascer, categorizado em três grupos, o ajuste do modelo foi realizado utilizando-se a regressão logística multinomial, tendo como categoria base ao grupo de 2.500 a $4.000 \mathrm{~g}$. A regressão foi de tipo "backward stepwise" e o critério para permanência no modelo foi de $\mathrm{p} \leq 0,05$. Em todas as análises, foi adotado valor $\mathrm{p}<0,05$ de um teste bicaudal. Os cálculos da análise ajustada foram realizados no programa Stata 11.

O protocolo de pesquisa foi submetido e aprovado pelo Comitê de Ética em Pesquisa na Área da Saúde (CEPAS) da FURG. Além disso, garantiu-se a confidencialidade dos dados, a participação voluntária e a possibilidade de deixar o estudo a qualquer momento, sem necessidade de justificativa.

\section{Resultados}

Das 1.235 puérperas incluídas, 53,6\% apresentavam idade entre 20 e 29 anos; 65,9\% eram de cor branca; 82,9\% tinham companheiro; $46,9 \%$ referiam ter 4 anos ou menos de estudo e $74,4 \%$ relatavam renda familiar superior ou igual a 1 salário-mínimo. Em relação ao pré-natal, a cobertura encontrada foi de $100 \%$ nesse grupo.

Quanto aos dados referentes ao peso, 63,1\% das puérperas referiam ter peso entre 51 e $70 \mathrm{~kg}$ no início da gestação, com uma média de $63,6 \mathrm{~kg}$. Ao final do pré-natal, $51,4 \%$ das puérperas estavam com peso de igual ou superior a $71 \mathrm{~kg}$, com média de $73 \mathrm{~kg}$. O ganho de peso durante a gestação foi de até $12 \mathrm{~kg} \mathrm{em} \mathrm{70,9 \%} \mathrm{das} \mathrm{gestantes.} \mathrm{Entre} \mathrm{as}$ 1.117 entrevistadas, das quais foi possível calcular o IMC no início da gestação, 55,9\% estavam incluídas nos valores entre 18,5 e 24,9 (Tabela 1 ).

Tabela 1. Distribuição da amostra e cálculo do índice de massa corporal do início da gestação e do ganho de peso gestacional para avaliação do risco gestacional de hipertensão, diabetes, parto pré-termo, realização de cesárea e baixo peso ao nascer em estudo de base populacional

\begin{tabular}{|c|c|c|}
\hline & n & $\%$ \\
\hline \multicolumn{3}{|l|}{ Idade (anos) } \\
\hline 19 ou menos & 278 & 22,5 \\
\hline 20 a 29 & 662 & 53,6 \\
\hline 30 ou mais & 295 & 23,9 \\
\hline \multicolumn{3}{|l|}{ Cor da pele } \\
\hline Branca & 814 & 65,9 \\
\hline Não branca & 421 & 34,1 \\
\hline \multicolumn{3}{|l|}{ Escolaridade (anos) } \\
\hline 9 ou mais & 173 & 14,0 \\
\hline 5 a 8 & 483 & 39,1 \\
\hline 4 ou menos & 579 & 46,9 \\
\hline \multicolumn{3}{|l|}{ Renda per capita } \\
\hline <1 salário-mínimo & 316 & 25,6 \\
\hline Zl salário-mínimo & 919 & 74,4 \\
\hline \multicolumn{3}{|l|}{ Peso no início da gestação (kg) } \\
\hline Média \pm desvio padrão & $63,6 \pm 13,3$ & \\
\hline \multicolumn{3}{|l|}{ Peso no final da gestação (kg) } \\
\hline Média \pm desvio padrão & $73,1 \pm 13,9$ & \\
\hline \multicolumn{3}{|l|}{ Ganho de peso na gestação (kg) } \\
\hline 8 ou menos & 560 & 45,3 \\
\hline 9 a 12 & 316 & 25,6 \\
\hline 13 a 16 & 215 & 17,4 \\
\hline 17 ou mais & 144 & 11,7 \\
\hline Média \pm desvio padrão & $9,4 \pm 5,6$ & \\
\hline IMC no início da gestação $\left(\mathrm{kg} / \mathrm{m}^{2}\right)$ & 1.117 & \\
\hline 18,4 ou menos & 42 & 3,9 \\
\hline 18,5 a 24,9 & 595 & 55,9 \\
\hline 25 a 29,9 & 289 & 23,2 \\
\hline 30 ou mais & 138 & 13,0 \\
\hline Média \pm desvio padrão & $24,7 \pm 4,7$ & \\
\hline
\end{tabular}

IMC: Índice de massa corporal (peso/altura²). 
Tabela 2. Análise ajustada do risco gestacional para hipertensão, diabetes, parto pré-termo, realização de cesárea e baixo peso ao nascer em relação ao índice de massa corporal no início do pré-natal em estudo de base populacional

\begin{tabular}{|c|c|c|c|c|c|}
\hline & \multicolumn{4}{|c|}{ IMC (início da gestação) } & \multirow[b]{2}{*}{ Valor $p$} \\
\hline & $\begin{array}{l}18,4 \text { ou menos } \\
\text { Odds Ratio }\end{array}$ & $\begin{array}{c}18,5 \text { a } 24,9 \\
\text { Odds Ratio (IC95\%) }\end{array}$ & $\begin{array}{c}25 \text { a } 29,9 \\
\text { Odds Ratio (IC95\%) }\end{array}$ & $\begin{array}{c}30 \text { ou mais } \\
\text { Odds Ratio (IC95\%) }\end{array}$ & \\
\hline Hipertensão & 1,0 & $1,1(1,0-1,1)$ & $0,9(0,9-1,0)$ & $1,0(0,9-1,2)$ & $0,003^{\star}$ \\
\hline Parto pré-termo & 1,0 & $0,9(0,9-1,1)$ & $0,9(0,9-1,1)$ & $0,9(0,9-1,1)$ & $0,9^{*}$ \\
\hline Parto cesárea & 1,0 & $1,0(0,9-1,1)$ & $1,1(0,9-1,2)$ & $1,1(0,9-1,2)$ & $0,004^{\star \star}$ \\
\hline$\geq 4.000$ & 1,0 & $1,7(0,2-13,1)$ & $3,4(0,4-26,1)$ & $6,7(0,9-52,5)$ & $0,001^{\star * \star}$ \\
\hline
\end{tabular}

IMC: índice de massa corporal; IC95\%: intervalo de confiança de 95\%.

*Teste de heterogeneidade; **teste de tendência linear; *** regressão logística multinominal ordinal com categoria de base de $2.500 \mathrm{a} 4.000 \mathrm{~g}$.

Tabela 3. Análise ajustada do risco gestacional para hipertensão, diabetes, parto pré-termo, realizacão de cesárea e baixo peso ao nascer em relacão ao ganho de peso na gestacão em estudo de base populacional

\begin{tabular}{|c|c|c|c|c|c|}
\hline & \multicolumn{4}{|c|}{ Ganho de peso na gestação (kg) } & \multirow[b]{2}{*}{ Valor $p$} \\
\hline & $\begin{array}{l}8 \text { ou menos } \\
\text { Odds Ratio }\end{array}$ & $\begin{array}{c}9 \text { a } 12 \\
\text { Odds Ratio (IC95\%) }\end{array}$ & $\begin{array}{c}13 \text { a } 16 \\
\text { Odds Ratio (IC95\%) }\end{array}$ & $\begin{array}{c}17 \text { ou mais } \\
\text { Odds Ratio (IC95\%) }\end{array}$ & \\
\hline Hipertensão/diabetes & 1,0 & $1,0(0,9-1,0)$ & $1,0(0,9-1,1)$ & $0,9(0,9-1,0)$ & $0,7^{\star}$ \\
\hline Parto cesárea & 1,0 & $0,9(0,9-1,0)$ & $0,9(0,9-1,0)$ & $0,9(0,9-1,0)$ & $0,05^{\star}$ \\
\hline Peso ao nascer*** $(g)$ & 1,0 & $1,1(1,0-1,1)$ & $1,1(1,0-1,2)$ & $1,1(1,1-1,2)$ & $<0,001^{\star \star}$ \\
\hline$\geq 4.000$ & 1,0 & $1,3(0,7-2,4)$ & $1,1(0,6-2,3)$ & $1,7(0,8-3,4)$ & $0,03^{\star \star \star}$ \\
\hline
\end{tabular}

IC95\%: intervalo de confiança de 95\%.

*Teste de heterogeneidade; **teste de tendência linear; *** regressão logística multinominal com categoria de base de 2.500 a $4.000 \mathrm{~g}$

Em relação ao risco gestacional e o valor do IMC no início da gestação, podemos observar que o grupo com IMC entre 18,5 e 24,9 apresentou maior risco de ter hipertensão quando comparado aos os outros grupos. Quanto ao risco de parto cesárea observou-se que quanto maior o IMC no início da gestação, maior o risco de parto cirúrgico, sendo maior no grupo de $I M C \geq 30(p=0,004)$. Outra variável influenciada pelo IMC gestacional foi o peso do recém-nascido, quando fica evidente que quanto maior o IMC no início da gestação, menor o risco de baixo peso ao nascer $(\mathrm{p}=0,007)$ e maior o risco de macrossomia $(\mathrm{p}=0,001)$. Não foi observado aumento na incidência de diabetes e risco de trabalho de parto pré-termo nas pacientes dos diferentes grupos de IMC (Tabela 2).

$\mathrm{Na}$ Tabela 3 tem-se o desfecho gestacional em relação ao ganho de peso na gestação e pode-se observar que tanto o desenvolvimento de hipertensão como o de diabetes não apresentaram associação significante com ganho ponderal. No entanto, o risco de trabalho de parto prematuro foi maior no grupo de mulheres que apresentavam um ganho de peso $\leq 8 \mathrm{~kg}(\mathrm{p}<0,05)$. Em relação à via de parto, observa-se que quanto maior o ganho de peso durante a gestação, maior o risco de parto cirúrgico, sendo maior no grupo com ganho $\geq 17 \mathrm{~kg}(\mathrm{p}=0,001)$.
O peso do recém-nascido também foi influenciado pelo ganho de peso materno, sendo evidenciado que quanto maior o ganho de peso gestacional, menor o risco de baixo peso ao nascer $(p=0,01)$ e maior o risco de macrossomia, principalmente no grupo com ganho $\geq 17 \mathrm{~kg}(\mathrm{p}=0,03)$.

\section{Discussão}

O sobrepeso e a obesidade apresentam uma prevalência elevada em todo o mundo ${ }^{23}$. Um estudo retrospectivo realizado com mulheres inglesas demonstrou um aumento nas taxas de obesidade de $9,9 \%$, em 1990, para $16 \%$ em 2004 , com projeção para $22 \%$ em $2010^{24}$. Admite-se que 19 a $38 \%$ das gestações ocorrem em mulheres com sobrepeso ou obesas ${ }^{25}$. No presente estudo pode-se observar que $23,2 \%$ das gestantes apresentavam sobrepeso no início da gestação e $13 \%$ eram obesas. No Brasil, resultados semelhantes foram encontrados em estudo realizado pelo Núcleo de Pesquisa em Nutrição e Saúde da Universidade de São Paulo (NUPENS/USP), no qual $27 \%$ apresentavam-se com sobrepeso/obesidade ${ }^{26}$. Outro parâmetro avaliado por este estudo foi o ganho de peso gestacional, sendo em média de $9,4 \mathrm{~kg}$, com um ganho excessivo de peso presente em $29,1 \%$ das gestantes. Estudos brasileiros com gestantes da rede básica de saúde 
encontraram uma incidência de ganho ponderal excessivo de $29 \%$ e insuficiente de 36,5 a $38 \%{ }^{25,27-29}$.

A inadequação do estado antropométrico materno, tanto pré-gestacional como gestacional, constitui um problema de saúde pública inquestionável, pois favorece o desenvolvimento de intercorrências gestacionais e influencia nas condições de saúde materna e do concepto, no período pós-parto ${ }^{7,14,15}$. Estudo de caso-controle realizado no Hospital de Clínicas da Universidade Federal de Goiás (HC-UFG) verificou que sobrepeso/obesidade durante a gravidez estão associados à hipertensão gestacional, pré-eclâmpsia e hipertensão arterial crônica, e que o risco de pré-eclâmpsia duplica para cada 5 a $7 \mathrm{~kg} / \mathrm{m}^{2}$ de aumento no IMC no início da gestação ${ }^{17}$. Da mesma forma, vários estudos associam o ganho de peso excessivo na gestação a um maior risco de desenvolver hipertensão, diabete, parto cirúrgico e obesidade ${ }^{9,20-22}$.

A literatura associa sobrepeso/obesidade pré-gestacional e ganho ponderal excessivo na gestação com o risco de desenvolvimento de hipertensão e diabete, mas esta associação não foi encontrada neste estudo. Estes achados podem estar associados ao não diagnóstico destas morbidades entre as gestantes estudadas visto que a hipertensão e a hemorragia ainda são as principais causa de óbito materno no Brasil ${ }^{30,31}$. Estudo anterior realizado no município do Rio Grande observou que, apesar da cobertura pré-natal ser de $95,8 \%$ e a média de consultas ser de 7,4 consultas/gestante, quando se avalia a qualidade da atenção prestada, apenas $26,8 \%$ tem seu pré-natal classificado como adequado ${ }^{32}$.

Outro parâmetro do desfecho gestacional avaliado neste estudo foi a presença de parto pré-termo. O IMC pré-gestacional não influenciou no risco de trabalho de parto pré-termo, mas observou-se associação entre ganho ponderal e prematuridade. Foi observado que as gestantes com um ganho de peso $\leq 8 \mathrm{~kg}$ durante a gestação apresentaram maior risco de parto pré-termo. No Brasil, a partir da década de 1990, ocorreu o aumento da frequência de prematuridade que passou de 5\%, em 1994, para 6,5\% em $2004^{18}$. Entre os fatores de risco associados ao parto prematuro, estão o baixo peso materno pré-gestacional e $\mathrm{o}$ insuficiente ganho de peso materno ${ }^{10,18,19,33}$.

A prevalência de cesariana na população estudada foi de $53,2 \%$, sendo muito superior aos $15 \%$ preconizado pela OMS. O Brasil apresenta uma das maiores taxas mundiais de parto cesárea, segundo o Sistema de Informações de Nascidos Vivos (SINASC), a taxa de cesariana no país aumentou de 39\%, em 1994, para 44\% em $2005^{34}$. Em nosso estudo, a via de parto apresentou relação direta com IMC pré-gestacional e o ganho de peso durante a gestação. As mulheres com sobrepeso/ obesidade e com ganho ponderal $>9 \mathrm{~kg}$ apresentaram um maior risco de ter o parto por via cirúrgica. Vários autores correlacionam sobrepeso/obesidade gestacional com o risco aumentado de parto cesárea entre as principais indicações para este procedimento está a desproporção céfalo-pélvica (DCP), síndromes hipertensivas e diabetes $^{6,10,14,15,19,35}$. Estudo realizado por Seligman et al. ${ }^{15}$ demonstrou que as mulheres obesas tiveram uma maior taxa de indicação de cesariana, e que todas as indicações relatadas, exceto apresentação anômala, foram superiores em pacientes obesas quando comparada com gestantes eutróficas. Para estes pesquisadores, as mulheres obesas são mais suscetíveis ao trabalho de parto prolongado, devido a seus maiores tecidos moles pélvicos, resultando em um canal de parto estreito. Assim, a cesariana acaba sendo recomendada nessas mulheres, a fim de prevenir estresse fetal.

Quanto ao peso do recém-nascido ao nascer, foi constatado, em nosso estudo, que mulheres que iniciam a gestação desnutridas ou que apresentam ganho de peso insuficiente, tem risco mais elevado de terem recém-nascidos com peso inferior a $2.500 \mathrm{~g}$. Em contrapartida, os fetos de mulheres com sobrepeso/obesidade no início da gestação ou com maior ganho de peso tendem a macrossomia. Vários estudos associam desnutrição materna a uma maior prevalência de restrição de crescimento intrauterino e baixo peso ao nascer (PIG), sendo a desnutrição crônica mais importante que o ganho ponderal insuficiente durante o período gestacional gestacional ${ }^{12,13}$. Por outro lado, a obesidade tem um impacto significativo sobre o metabolismo dos macronutrientes, alterando a síntese de aminoácidos, resultando em uma possível interferência no desenvolvimento do concepto. Desta forma, a obesidade materna e o ganho de peso excessivo podem estar associados à macrossomia e/ou obesidade futura para a criança ${ }^{8,13,28,36}$. Estudo realizado no Rio de Janeiro, com 230 gestantes, revelou que mulheres com ganho de peso excessivo na gestação apresentam 5,83 vezes mais chances de dar à luz uma criança com macrossomia ${ }^{28}$.

Podemos concluir que tanto o sobrepeso/obesidade pré-gestacional como o ganho de peso insuficiente ou excessivo durante a gestação têm implicações diretas sobre o risco gestacional e o seu desfecho. Além disso, estudos associam o ganho de peso excessivo na gestação a um maior risco de desenvolver sobrepeso/obesidade nos próximos 15 $\operatorname{anos}^{35,37}$. Os médicos clínicos ou pré-natalistas devem estar preparados para orientar as mulheres da importância da redução do peso antes do início da gravidez e também de como evitar o excesso de ganho de peso na gestação. Para que isto seja realizado, a avaliação do IMC deve ser feita tanto nas consultas clínicas, como na primeira consulta de pré-natal. O cálculo do IMC, além de ser um instrumento que auxilia no desenvolvimento de intervenções precoces, apresenta baixo custo de realização. 
1. Stefan N, Kantartzis K, Machann J, Schick F, Häring HU. Global trends in body-mass index. Lancet. 2011;377(9781):1917.

2. Morabia A, Costanza MC. The obesity epidemic as harbinger of a metabolic disorder epidemic: trends in overweight, hypercholesterolemia, and diabetes treatment in Geneva, Switzerland, 1993-2003. Am J Public Health. 2005;95(4):632-5.

3. Marques-Vidal P, Dias CM. Trends in overweight and obesity in Portugal: the National Health Surveys 1995-6 and 1998-9. Obes Res. 2005;13(7):1141-5.

4. Mendez MA, Monteiro CA, Popkin BM. Overweight exceeds underweight among women in most developing countries. Am J Clin Nutr. 2005;81 (3):714-21.

5. Dong HJ, Unosson M, Wressle E, Marcusson J. Health consequences associated with being overweight or obese: a Swedish populationbased study of 85-year-olds. J Am Geriatr Soc. 2012;60(2):243-50.

6. Yazdani S, Yosofniyapasha Y, Nasab BH, Mojaveri MH, Bouzari $Z$. Effect of maternal body mass index on pregnancy outcome and newborn weight. BMC Res Notes. 2012;5:34.

7. Dodd JM, Turnbull DA, McPhee AJ, Wittert G, Crowther CA, Robinson JS. Limiting weight gain in overweight and obese women during pregnancy to improve health outcomes: the LIMIT randomised controlled trial. BMC Pregnancy Childbirth. 2011;11:79.

8. Persson $M$, Pasupathy $D$, Hanson $U$, Westgren $M$, Norman $M$. Pre-pregnancy body mass index and the risk of adverse outcome in type 1 diabetic pregnancies: a population-based cohort study. BM Open. 2012;2(1):e000601.

9. Davies GA, Maxwell C, McLeod L, Gagnon R, Basso M, Bos H, et al. Obesity in pregnancy. J Obstet Gynaecol Can. 2010;32(2): 165-73.

10. Siega-Riz AM, Laraia B. The implications of maternal overweight and obesity on the course of pregnancy and birth outcomes. Matern Child Health J. 2006; 10(5 Suppl):S153-6.

11. Machado LS. Cesarean section in morbidly obese parturients: practical implications and complications. N Am J Med Sci. 2012;4(1):13-8.

12. Assunção PL, Mello AS, Gondim SS, Benício MH, Amorim MM, Cardoso AM. Ganho ponderal e desfechos gestacionais em mulheres atendidas pelo Programa de Saúde da Família em Campina Grande, PB (Brasil). Rev Bras Epidemiol. 2007;10(3):352-60.

13. Konno SC, Benício MH, Barros AJ. Fatores associados à evolução ponderal de gestantes: uma análise multinível. Rev Saúde Pública. 2007;41(6):995-1002

14. Seabra G, Padilha PC, Queiroz JA, Saunders C. [Pregestational overweight and obesity: prevalence and outcome associated with pregnancy]. Rev Bras Ginecol Obstet. 2011 ; 33(1 1):348-53.Portuguese.

15. Seligman LC, Duncan BB, Branchtein L, Gaio DS, Mengue SS, Schmidt Ml. Obesity and gestational weight gain: cesarean delivery and labor complications. Rev Saúde Pública. 2006;40(3):457-65.

16. Madi JM, Rombaldi RL, Oliveira Filho PF, Araújo BF, Zatti H, Madi SR. [Maternal and perinatal factors related to fetal macrossomia]. Rev Bras Ginecol Obstet. 2006;28(4):232-7. Portuguese.

17. Assis TR, Viana FP, Rassi S. Estudo dos principais fatores de risco maternos nas síndromes hipertensivas da gestação. Arq Bras Cardiol. 2008;91(1):11-7.

18. Silveira MF, Santos IS, Barros AJ, Matijasevich A, Barros FC, Victora CG. Aumento da prematuridade no Brasil: revisão de estudos de base populacional. Rev Saúde Pública. 2008;42(5):957-64.

19. Whiteman VE, Crisan L, McIntosh C, Alio AP, Duan J, Marty PJ, et al. Interpregnancy body mass index changes and risk of stillbirth. Gynecol Obstet Invest. $2011 ; 72(3): 192-5$.
20. World Health Organization [Internet]. Global database on body mass index: an interactive surveillance tool for monitoring nutrition transition. 2012 [cited 2012 May 10]. Available from: <http:// apps.who.int/bmi/index.jsp>

21. Blomberg M. Maternal and neonatal outcomes among obese women with weight gain below the new Institute of Medicine recommendations. Obstet Gynecol. 2011;117(5):1065-70.

22. Brasil. Ministério da Saúde. Secretaria de Atenção à Saúde. Departamento de Ações Programáticas Estratégicas. Área Técnica de Saúde da Mulher. Pré-natal e puerpério: atenção qualificada e humanizada: manual técnico. Brasília (DF): Ministério da Saúde; 2005.

23. Wax JR. Risks and management of obesity in pregnancy: current controversies. Curr Opin Obstet Gynecol. 2009;21 (2): 1 17-23.

24. Heslehurst N, Ells L, Simpson H, Batterham A, Wilkinson J, Summerbell CD. Trends in maternal obesity incidence rates, demographic predictors, and health inequalities in 36,821 women over a 15-year period. BJOG. 2007; 1 14(2): 187-94.

25. Hickey $C A$. Sociocultural and behavioral influences on weight gain during pregnancy. Am J Clin Nutr. 2000;71 (5 Suppl): 1364S-70S.

26. Mello AS, Assunção PL, Gondim SS, Carvalho DF, Amorim MM, Benicio $\mathrm{MH}$, et al. Estado nutricional materno, ganho de peso gestacional e peso ao nascer. Rev Bras Epidemiol. 2007; 10(2):249-57.

27. Bodnar LM, Catov JM, Klebanoff MA, Ness RB, Roberts JM. Prepregnancy body mass index and the occurrence of severe hypertensive disorders of pregnancy. Epidemiology. 2007;18(2):234-9.

28. Kac $G$, Velásquez-Meléndez $G$. Ganho de peso gestacional e macrossomia em uma coorte de mães e filhos. J Pediatr (Rio J). 2005;81 (1):47-53.

29. Ota E, Haruna M, Suzuki M, Anh DD, Tho le $H$, Tam NT, et al. Maternal body mass index and gestational weight gain and their association with perinatal outcomes in Viet Nam. Bull World Health Organ. $2011 ; 89(2): 127-36$.

30. Peraçoli JC, Parpinelli MA. [Hypertensive disorders of pregnancy: identifying severe cases]. Rev Bras Ginecol Obstet. 2005;27(10):627. 34. Portuguese.

31. Sass $N$, Itamoto $C H$, Silva MP, Torloni MR, Atallah AN. Does sodium nitroprusside kill babies? A systematic review. Sao Paulo Med J. 2007;125(2):108-11.

32. Gonçalves CV, Cesar JA, Mendoza-Sassi RA. Qualidade e eqüidade na assistência a gestante: um estudo de base populacional no Sul do Brasil. Cad Saúde Pública. 2009;25(1 1):2507-16.

33. Santos IS, Matijasevich A, Silveira MF, Sclowitz IK, Barros A, Victora CG, et al. Associated factors and consequences of late preterm births: results from the 2004 Pelotas birth cohort. Paediatr Perinat Epidemiol. 2008;22(4):350-9.

34. Brasil. Ministério da Saúde. Centro Brasileiro de Análise e Planejamento. Pesquisa Nacional de Demografia e Saúde da Criança e da Mulher - PNDS 2006: dimensões do processo reprodutivo e da saúde da criança. Brasília (DF): Ministério da Saúde; 2009.

35. Mamun AA, Callaway LK, O'Callaghan M, Williams GM, Najman $J M$, Alati R, et al. Associations of maternal pre-pregnancy obesity and excess pregnancy weight gains with adverse pregnancy outcomes and length of hospital stay. BMC Pregnancy Childbirth. $2011 ; 11: 62$.

36. Luoto R, Kinnunen TI, Aittasalo M, Kolu P, Raitanen J, Ojala K, et al. Primary prevention of gestational diabetes mellitus and large-for-gestational-age newborns by lifestyle counseling: a clusterrandomized controlled trial. PLoS Med. 201 1;8(5):e1001036.

37. Linné Y, Dye L, Barkeling B, Rössner S. Long-term weight development in women: a long 15-year follow-up of the effects of pregnancy. Obest Res. 2004;12(7):1166-78. 